\title{
HALF-AXES IN POWER ASSOCIATIVE ALGEBRAS
}

\author{
YOAV SEGEV
}

\begin{abstract}
Let $A$ be a commutative, non-associative algebra over a field $\mathbb{F}$ of characteristic $\neq 2$. A half-axis in $A$ is an idempotent $e \in A$ such that $e$ satisfies the Peirce multiplication rules in a Jordan algebra, and, in addition, the 1-eigenspace of $\operatorname{ad}_{e}$ (multiplication by $e$ ) is one dimensional.

In this paper we consider the identities

(*) $x^{2} x^{2}=x^{4}$ and $x^{3} x^{2}=x x^{4}$.

We show that if identities $(*)$ hold strictly in $A$, then one gets (very) interesting identities between elements in the eigenspaces of $\operatorname{ad}_{e}$ (note that if $|\mathbb{F}|>3$ and the identities $(*)$ hold in $A$, then they hold strictly in $A$ ). Furthermore we prove that if $A$ is a primitive axial algebra of Jordan type half (i.e., $A$ is generated by half-axes), and the identities (*) hold strictly in $A$, then $A$ is a Jordan algebra.
\end{abstract}

\section{INTRODUCTION}

Throughout this paper $\mathbb{F}$ is a field of characteristic not 2 and $A$ is a commutative non-associative algebra over $\mathbb{F}$. Given an element $x \in A$ and a scalar $\lambda \in \mathbb{F}$, we denoted:

$$
A_{\lambda}(x):=\{y \in A \mid y x=\lambda y\} .
$$

(We allow $A_{\lambda}(x)=0$.)

Definition 1.1. Let $e \in A$, and set $Z:=A_{0}(e)$ and $U:=A_{1 / 2}(e)$. We say that $e$ is a half-axis if and only if

(1) $e^{2}=e$ (so $e$ is an idempotent).

(2) $A_{1}(e)=\mathbb{F} e$.

(3) $A=\mathbb{F} e \oplus U \oplus Z$.

(4) $Z^{2} \subseteq Z, U^{2} \subseteq \mathbb{F} e+Z$ and $U Z \subseteq U$.

Note that any idempotent $e$ in a Jordan algebra $J$ such that $J_{1}(e)=\mathbb{F} e$ is a half-axis.

Recall that $A$ is a primitive axial algebra of Jordan type half if $A$ is generated (as an algebra over $\mathbb{F}$ ) by half-axes.

We also need the following notation.

Date: Jan. 22, 2018.

2010 Mathematics Subject Classification. Primary: 17A05; Secondary: 17C99, 17B69.

Key words and phrases. half-axis, power associative algebra, Axial algebra, Jordan algebra. 
Notation 1.2. Let $e \in A$ be a half-axis, and let $x \in A$. Write $x=\alpha e+$ $x_{0}+x_{1 / 2}$, with $\alpha \in \mathbb{F}$ and $x_{\lambda} \in A_{\lambda}(e)$, for $\lambda \in\{0,1 / 2\}$.

(1) We denote $\varphi_{e}(x)=\delta_{x}:=\alpha$.

(2) We denote $z_{x}:=x_{0}$. We call $z_{x}$ the $Z$-part of $x$.

Note that $e x=\delta_{x} e$, for $x \in A_{1}(e)+A_{0}(e)$.

Throughout this paper we shall use the technique of linearization of identities. More details about this technique are given in $\S 2$.

1.3. Scalar extension and strict validity of identities. For a field extension $\mathbb{K} / \mathbb{F}$, we denote by $A_{\mathbb{K}}:=A \otimes_{\mathbb{F}} \mathbb{K}$ the scalar extension (or base change) of $A$ from $\mathbb{F}$ to $\mathbb{K}$, which is a commutative non-associative $\mathbb{K}$-algebra in a natural way. It is well known that Jordan algebras are invariant under base change (see e.g. [M, Linearization Proposition 1.8.5(2), p. 148]), so $A$ is a Jordan algebra over $\mathbb{F}$ if and only if $A_{\mathbb{K}}$ is one over $\mathbb{K}$. Moreover, since tensor products commute with direct sums, if $e \in A$ is a half-axis, then $e$ is a half-axis in $A_{\mathbb{K}}$. Since primitive axial algebras of Jordan type half are spanned by half-axes (see [HRS, Corollary 1.2, p. 81]), it follows that primitive axial algebras are stable under base change as well. But powerassociative algebras are not. For this reason, the concept of strict powerassociativity comes in: $A$ is called strictly power-associative if the scalar extensions $A_{\mathbb{K}}$ are power-associative, for all field extensions $\mathbb{K} / \mathbb{F}$. Similarly, an identity is said to hold strictly in $A$ if it is satisfied not only by $A$ but by all its scalar extensions.

Our main result is the following theorem.

Main Theorem. Let $A$ be primitive axial algebra of Jordan type half generated over $\mathbb{F}$ (as an algebra) by a set $\mathcal{A}$ of half-axes. The following are equivalent.

(i) $A$ is a Jordan algebra.

(ii) $A$ is strictly power associative.

(iii) The identities

$$
x^{2} x^{2}=x x^{3} \quad \text { and } \quad x^{3} x^{2}=x x^{4}
$$

hold strictly in $A$.

(iv) For all $e \in \mathcal{A}, u \in A_{1 / 2}(e)$ and $z \in A_{0}(e)$ we have

(a) $u^{3}=\delta_{u^{2}} u$.

(b) $(u z) z=\frac{1}{2} u z^{2}$.

The interesting implications of the Main Theorem are of course $(i i i) \Rightarrow$ $(i v)$ and $(i v) \Rightarrow(i)$. The implication $(i i i) \Rightarrow(i v)$ is part of Theorem 1.4 below, and the implication $(i v) \Rightarrow(i)$ is proven in $\S 3$.

Remark 1.3. Throughout this paper we will deal with the identities

$$
x^{2} x^{2}=x x^{3} \quad \text { and } \quad x^{3} x^{2}=x x^{4} .
$$


Note that if $|\mathbb{F}|>3$ and the identities of equation (1.1) hold in $A$, then, by Corollary 2.3 (2) below, these identities hold strictly in $A$. Hence if $|\mathbb{F}|>3$, and $A$ is a primitive axial algebra of Jordan type half such that the identities of equation (1.1) hold in $A$, then all the equivalent properties of the Main Theorem hold in $A$.

Theorem 1.4. Assume that the identities $x^{2} x^{2}=x x^{3}$ and $x^{3} x^{2}=x x^{4}$ hold strictly in $A$, and let $e \in A$ be a half-axis. Let $u \in A_{1 / 2}(e)$ and $z \in A_{0}(e)$. Then

(1) $u^{3}=\delta_{u^{2}} u$.

(2) $(u z) z=\frac{1}{2} u z^{2}$.

(3) $4 u^{2}(u z)=2 u(u(u z))+u\left(u^{2} z\right)+u^{3} z$.

(4) $4(u z)^{2}+2 u^{2} z^{2}=u\left(u z^{2}\right)+2(u(u z)) z+2 u((u z) z)+\left(u^{2} z\right) z$.

(5) $4(u z) z^{2}=u z^{3}+\left(u z^{2}\right) z+2((u z) z) z$.

(6) $z_{u(u z)}=\frac{1}{2} u^{2} z$.

Theorem 1.4 is proven in $\S 2$.

Remark 1.5. When $|\mathbb{F}|=3$, and the identities of equation (1.1) hold in $A$, one can show that identities (1), (4) and (6) of Theorem 1.4 remain valid. It is quite possible that additional (long) calculations will show that the other identities are valid as well. Once this is done it will follow from the implication $(i v) \Rightarrow(i)$ of the Main Theorem that if $A$ is a primitive axial algebra of Jordan type half and the identities of equation (1.1) hold in $A$, then $A$ is a Jordan algebra. (Indeed, by Remark 1.3 , if $|\mathbb{F}|>3$ and the identities of equation (1.1) hold in $A$, then they hold strictly in $A$, so Theorem 1.4 applies, and then the Main Theorem applies.)

We mention that Theorem 7.1 in $\S 7$ gives interesting necessary and sufficient conditions for a commutative non-associative algebra $A$ having a halfaxis to be a Jordan algebra. See also Theorem 7.2. Furthermore, throughout this paper we obtain additional various interesting identities.

Notation 1.6. Throughout this paper we let $e$ be a fixed half-axis in $A$. We denote

$$
U:=A_{1 / 2}(e) \text { and } Z=A_{0}(e) .
$$

To conclude the introduction we would like to mention that this paper was inspired by [TB].

\section{The Proof of Theorem 1.4}

In this section we adopt the notation and terminology of 0 . Thus we consider non-associative and non-commutative polynomials $f\left(x_{1}, \ldots, x_{m}\right)$ over $\mathbb{F}$. The degree of $x_{i}$ in each monomial of $f$ is defined on p. 167 of $\mathrm{O}$ and $f$ is homogeneous if, for each $i \in\{1, \ldots, m\}$, the degree of $x_{i}$ is the same in all of the monomials of $f$. 
Recall also from p. 176 of $[\mathrm{O}$ ] the notion of linearization of $f$ and the notion of a stable derivative of $f$. Note that all stable derivatives of $f$ contain all linearizations of $f$. A homogeneous identity is an identity $f\left(x_{1}, \ldots, x_{m}\right)=0$, where $f$ is a homogeneous polynomial. We will not distinguish between the polynomial $f$ and the identity $f=0$.

Corollary 2.3 below is well known. However, since we did not find an explicit reference to it, we include a proof. First we quote the following two results from $\mathrm{O}$.

Theorem 2.1 (Theorem 3.5 in $[\mathrm{O}]$ ). Let $\mathbb{L}$ be a field with $|\mathbb{L}| \geq d$, let $f\left(x_{1}, \ldots, x_{m}\right)$ be a homogeneous identity over $\mathbb{L}$, such that the degree of each $x_{i}, i=1, \ldots, m$, in $f$ is no more than $d$. Let $A$ be a $\mathbb{L}$-algebra satisfying $f$. Then A satisfies all stable derivatives of $f$.

Proposition 2.2. Let $S$ be a set of homogeneous identities over a field $\mathbb{K}$, and let $V$ be the variety of $\mathbb{K}$-algebras determined by $S$. Let $A \in V$ satisfy every stable derivative of each identity in $S$. Then, $A_{\mathbb{L}} \in V_{\mathbb{L}}$, for every field extension $\mathbb{L} / \mathbb{K}$, where $V_{\mathbb{L}}$ is the variety of $\mathbb{L}$-algebras determined by $S$.

Proof. See [O, Proposition 4.2].

Corollary 2.3. Let $f\left(x_{1}, \ldots, x_{m}\right)=0$ be a homogeneous identity over a field $\mathbb{K}$. Then

(1) $f$ holds strictly in $A$ if and only if $A$ satisfies all stable derivatives of $f$.

(2) If the degree of each $x_{i}, i=1, \ldots m$, in $f$ is at most $d$, and $|\mathbb{F}| \geq d$, then $f$ holds strictly in $A$.

Proof. (1): Suppose $f$ holds strictly in $A$. Let $\mathbb{L} / \mathbb{K}$ be an infinite field extension. Then $\mathbb{F}$ holds in $A_{\mathbb{L}}$, and $\mathbb{L}$ satisfies the hypothesis of Therem 2.1. Hence $A_{\mathbb{L}}$ satisfies every stable derivative of $f$. Since $A$ embeds in $A_{\mathbb{L}}$, $A$ satisfies every stable derivative of $f$.

Conversely, suppose $A$ satisfies all stable derivatives of $f$. Then by Proposition [2.2, $f$ holds strictly in $\mathbb{K}$.

(2): Assume the hypotheses of (2). By Theorem 2.1, $A$ satisfies every stable derivative of $f$. Hence (2) follows from (1).

In this section we prove Theorem 1.4, The proof uses linearization techniques. We repeatedly use Corollary 2.3 without further reference.

Lemma 2.4. Assume that the identity $x^{2} x^{2}=x^{3} x$ holds strictly in $A$ (in particular this holds if $|\mathbb{F}|>3$ ). Then the following identities hold in $A$

$$
\begin{gathered}
4 x^{2}(x y)=x^{3} y+x\left(x^{2} y\right)+2 x(x(x y)) . \\
8(x y)(x w)+4 x^{2}(y w)=\left(x^{2} y\right) w+\left(x^{2} w\right) y+2(x(x y)) w+2(x(x w)) y \\
+2 x((x y) w)+2 x((x w) y)+2 x((x(y w)) .
\end{gathered}
$$


Proof. Linearizing the identity $x^{2} x^{2}=x^{3} x$ at $x$ in the direction $y$ we get

$$
\left(x^{2}+2 x y+y^{2}\right)\left(x^{2}+2 x y+y^{2}\right)=\left[\left(x^{2}+2 x y+y^{2}\right)(x+y)\right](x+y) .
$$

Equating only the expressions in which $y$ is linear we get $4 x^{2}(x y)=x^{3} y+$ $x\left(x^{2} y\right)+2 x(x(x y))$, which is equation (2.1).

Next, linearizing equation (2.1) at $x$ in the direction $w$ we get

$$
\begin{aligned}
& 4(x+w)^{2}((x+w) y) \\
& =(x+w)^{3} y+(x+w)\left((x+w)^{2} y\right)+2(x+w)((x+w)((x+w) y)),
\end{aligned}
$$

or

$$
\begin{aligned}
& 4\left(x^{2}+2 x w+w^{2}\right)(x y+w y) \\
& =\left(\left(x^{2}+2 x w+w^{2}\right)(x+w)\right) y+(x+w)\left(\left(x^{2}+2 x w+w^{2}\right) y\right) \\
& +2(x+w)((x+w)(x y+w y)) .
\end{aligned}
$$

Equating only the expressions in which $w$ is linear we get

$$
\begin{gathered}
4 x^{2}(y w)+8(x y)(x w)=\left[\left(x^{2} w\right) y+2(x(x w)) y\right]+\left[2 x((x w) y)+\left(x^{2} y\right) w\right] \\
+[2 x(x(w y))+2 x((x y) w)+2(x(x y)) w] .
\end{gathered}
$$

rearranging we get equation (2.2).

Corollary 2.5. Assume that the identity $x^{2} x^{2}=x^{3} x$ holds strictly in $A$ (in particular this holds if $|\mathbb{F}|>3)$. Then

(1) identities (3), (4) and (5) of Theorem 1.4 hold with u replaced by any $x \in A$ and $z$ replaced by any $y \in A$.

(2) Identities (1) and (6) of Theorem 1.4 hold in $A$.

Proof. (1): Replacing $u$ by $x$ and $z$ by $y$ in identity (3) of Theorem 1.4. we get identity (2.1). Similarly we get identity (5) of Theorem 1.4. Putting $w=y$ in identity (2.2) we get the more general version of identity (4) of Theorem 1.4.

(2): Letting $y=e$ and $x=u \in U$ in identity (2.1), we get $2 u^{3}=$ $\frac{1}{2} u^{3}+\frac{1}{2} \delta_{u^{2}} u+u^{3}$, and identity (1) of Theorem 1.4 follows.

Finally let $w=e, x=u \in U$ and $y=z \in Z$ in identity (2.2). Then $\left(x^{2} y\right) e=\left(x^{2} e\right) y=0$ and hence by identity (2.2) we get

$$
4 u(u z)=2 e(u(u z))+u^{2} z+u(u z)+u(u z) .
$$

It follows that $u^{2} z=2(u(u z)-e(u(u z)))$, this shows identity (6) of Theorem 1.4 .

Proposition 2.6. Assume that the identity $\left(x x^{2}\right) x^{2}=x\left(x^{2} x^{2}\right)$ holds strictly in $A$ (in particular this holds if $|\mathbb{F}|>3$ ). Then idenity (2) of Theorem 1.4 holds in $A$.

Proof. We write the identity $\left(x x^{2}\right) x^{2}=x\left(x^{2} x^{2}\right)$ as

$$
\left[x, x^{2}, x^{2}\right]=0,
$$


in terms of the (trilinear) associator $[x, y, z]=(x y) z-x(y z)$. Liniarizing identity (2.3) in the direction of $y$ we get

$$
\left[y, x^{2}, x^{2}\right]+2\left[x, x y, x^{2}\right]+2\left[x, x^{2}, x y\right]=0 .
$$

Next we claim that the identity

$$
\begin{aligned}
0 & =\left[x, y v, x^{2}\right]+\left[y, v x, x^{2}\right]+\left[v, x y, x^{2}\right]+\left[x, x^{2}, y v\right] \\
& +\left[y, x^{2}, v x\right]+\left[v, x^{2}, x y\right]+2[x, x y, x v]+2[x, x v, x y],
\end{aligned}
$$

holds in $A$. Indeed, linearizing identity (2.4) at $x$ in the direction $v$ we get

$$
\begin{gathered}
{\left[y,(x+v)^{2},(x+v)^{2}\right]+2\left[(x+v),(x+v) y,(x+v)^{2}\right]} \\
+2\left[(x+v),(x+v)^{2},(x+v) y\right]=0 .
\end{gathered}
$$

So

$$
\begin{gathered}
\left(2\left[y, x v, x^{2}\right]+2\left[y, x^{2}, x v\right]\right)+2\left(\left[x, v y, x^{2}\right]+2[x, x y, x v]+\left[v, x y, x^{2}\right]\right) \\
+2\left(\left[x, x^{2}, v y\right]+2[x, x v, x y]+\left[v, x^{2}, x y\right]\right)=0
\end{gathered}
$$

dividing by 2 and rearranging we get identity (2.5).

Next linearizing identity (2.5) at $x$ in the direction $w$ we get

$$
\begin{aligned}
0 & =\left[x+w, y v,(x+w)^{2}\right]+\left[y, v(x+w),(x+w)^{2}\right] \\
& +\left[v,(x+w) y,(x+w)^{2}\right]+\left[x+w,(x+w)^{2}, y v\right] \\
& +\left[y,(x+w)^{2}, v(x+w)\right]+\left[v,(x+w)^{2},(x+w) y\right] \\
& +2[x+w,(x+w) y,(x+w) v]+2[x+w,(x+w) v,(x+w) y] .
\end{aligned}
$$

Doing similar calculations and rearranging we get

$$
\begin{aligned}
0 & =\left[y, v w, x^{2}\right]+\left[v, w y, x^{2}\right]+\left[w, y v, x^{2}\right] \\
& +2[y, x v, x w]+2[v, x w, x y]+2[w, x y, x v] \\
& +2[y, x w, x v]+2[w, x v, x y]+2[v, x y, x w] \\
& +\left[y, x^{2}, v w\right]+\left[v, x^{2}, w y\right]+\left[w, x^{2}, y v\right] \\
& +2[x, y v, x w]+2[x, v w, x y]+2[x, w y, x v] \\
& +2[x, x y, v w]+2[x, x v, w y]+2[x, x w, y v] .
\end{aligned}
$$

Here we put $w=v=e$ and $x=z \in Z, y=u \in U$, to deduce

$$
\begin{aligned}
0 & =\left[u, e, z^{2}\right]+\left[e, e u, z^{2}\right]+\left[e, u e, z^{2}\right] \\
& +2[u, z e, z e]+2[e, z e, z u]+2[e, z u, z e] \\
& +2[u, z e, z e]+2[e, z e, z u]+2[e, z u, z e] \\
& +\left[u, z^{2}, e e\right]+\left[e, z^{2}, e u\right]+\left[e, z^{2}, u e\right] \\
& +2[z, u e, z e]+2[z, e e, z u]+2[z, e u, z e] \\
& +2[z, z u, e e]+2[z, z e, e u]+2[z, z e, u e] .
\end{aligned}
$$


So

$$
\begin{aligned}
0 & =\left[u, e, z^{2}\right]+2\left[e, e u, z^{2}\right] \\
& +0+0+0 \\
& +0+0+0 \\
& +\left[u, z^{2}, e\right]+\left[e, z^{2}, e u\right]+\left[e, z^{2}, u e\right] \\
& +0+2[z, e, z u]+0 \\
& +2[z, z u, e]+0+0 .
\end{aligned}
$$

The fusion rules now yield:

$$
0=\frac{1}{2} u z^{2}+0+\frac{1}{2} u z^{2}-\frac{1}{4} u z^{2}-\frac{1}{4} u z^{2}-z(z u)+0 .
$$

This shows that identity (2) of Theorem 1.4 holds.

Of course Theorem 1.4 follows from Corollary 2.5 and Proposition 2.6 .

\section{The proof of the Main Theorem}

In this section $A$ is a commutative non-associative algebra (at the moment we do not put any additional hypotheses on $A$ ). We now prove the Main Theorem.

The implication $(i) \Rightarrow(i i)$ is well known and follows from the fact that Jordan algebras are stable under base change and are power associative. The implication $(i i) \Rightarrow(i i i)$ is obvious, while the implication $(i i i) \Rightarrow(i v)$ follows from Theorem 1.4.

We now prove the implication $(i v) \Rightarrow(i)$. We start with:

Lemma 3.1. Let $u \in U$. Then the following are equivalent:

(i) $u^{3}=\delta_{u^{2}} u$.

(ii) $u z_{u^{2}}=\frac{1}{2} \delta_{u^{2}} u$.

(iii) $\left(e u^{2}\right) u=\frac{1}{2} u^{3}$.

Furthermore, if (i)-(iii) hold and $u^{2} u^{2}=u^{3} u$, then $z_{u^{2}}^{2}=\delta_{u^{2}} z_{u^{2}}$.

Proof. $(i) \Longleftrightarrow($ ii $)$ : We have

$$
u^{3}=u^{2} u=\left(\delta_{u^{2}} e+z_{u^{2}}\right) u=\frac{1}{2} \delta_{u^{2}} u+u z_{u^{2}} .
$$

Hence $(i)$ and $(i i)$ are equivalent.

$(i) \Longleftrightarrow(i i i):$ Since $\left(e u^{2}\right) u=\left(\delta_{u^{2}} e\right) u=\frac{1}{2} \delta_{u^{2}} u$ we see that $(i)$ and $(i i i)$ are equivalent.

Suppose that $u^{2} u^{2}=u^{3} u$ and that $(i)$ holds. Then

$$
\delta_{u^{2}}^{2} e+z_{u^{2}}^{2}=\left(u^{2}\right)^{2}=u^{3} u=\delta_{u^{2}} u^{2}=\delta_{u^{2}}^{2} e+\delta_{u^{2}} z_{u^{2}} .
$$

Lemma 3.2. Assume that $u^{3}=\delta_{u^{2}} u$ and that $(u z) z=\frac{1}{2} u z^{2}$, for all $u \in U$ and $z \in Z$. Then $x\left(x^{2} e\right)=x^{2}(x e)$, for all $x \in A$. 
Proof. Write $x=\alpha e+u+z$, with $u \in U$ and $z \in Z$. Then

$$
\begin{aligned}
x^{2} & =\alpha^{2} e+u^{2}+z^{2}+\alpha u+2 u z . \\
x e & =\alpha e+\frac{1}{2} u . \\
x^{2} e & =\alpha^{2} e+e u^{2}+\frac{1}{2} \alpha u+u z .
\end{aligned}
$$

Hence

$$
\begin{aligned}
& \left(x^{2} e\right) x=\left(\alpha^{2} e+e u^{2}+\frac{1}{2} \alpha u+u z\right)(\alpha e+u+z) \\
& =\alpha^{3} e+\frac{3}{4} \alpha^{2} u+\alpha e u^{2}+\frac{1}{2} u^{3}+\frac{1}{2} \alpha u^{2}+\alpha u z+u(u z)+(u z) z .
\end{aligned}
$$

Indeed $\frac{3}{4} \alpha^{2} u$ is obtained from $\left(\alpha^{2} e\right) u$ and $\left(\frac{1}{2} \alpha u\right)(\alpha e)$. And $\alpha u z$ is obtained from $\left(\frac{1}{2} \alpha u\right) z$ and $(u z)(\alpha e)$. Further, the equality $\left(e u^{2}\right) u=\frac{1}{2} u^{3}$ holds by hypothesis, and by Lemma 3.1 .

Next we compute

$$
\begin{aligned}
x^{2}(x e) & =\left(\alpha^{2} e+u^{2}+z^{2}+\alpha u+2 u z\right)\left(\alpha e+\frac{1}{2} u\right) \\
& =\alpha^{3} e+\alpha e u^{2}+\frac{3}{4} \alpha^{2} u+\alpha u z \\
& +\frac{1}{2} u^{3}+\frac{1}{2} u z^{2}+\frac{1}{2} \alpha u^{2}+u(u z) .
\end{aligned}
$$

Indeed $\frac{3}{4} \alpha^{2} u$ is obtained from $(\alpha u)(\alpha e)$ and $\left(\alpha^{2} e\right)\left(\frac{1}{2} u\right)$. Note now that all that remains to show in order to show that $\left(x^{2} e\right) x=x^{2}(x e)$, is the equality $(u z) z=\frac{1}{2} u z^{2}$. This equality holds by hypothesis.

We can now prove the implication $(i v) \Rightarrow(i)$ of the Main Theorem.

Suppose that $A$ is a primitive axial algebra of Jordan type half generated by a set of half-axes $\mathcal{A}$. By [HRS, Corollary 1.2, p. 81] (see also [HSS2, Corollary 3.4]) we may assume that $A$ is spanned by $\mathcal{A}$. Hence to show the identity $x^{2}(x y)=x\left(x^{2} y\right)$, for all $x, y \in A$, it suffices to show this identity when $y=f$ is an arbitrary half-axis $f \in \mathcal{A}$. Since $e$ is an arbitrary halfaxis in $A$, Lemma 3.2, and hypotheses $(i v)$ of the Main Theorem show that $x^{2}(x f)=x\left(x^{2} f\right)$, for all half-axes $f \in \mathcal{A}$.

\section{Consequences of Theorem 1.4}

In this section we prove some consequences of Theorem 1.4 which are useful to know and which will be applied in the following sections. Thus, throughout this section we assume that the identities $x^{2} x^{2}=x x^{3}$ and $x^{3} x^{2}=$ $x x^{4}$ hold strictly in $A$.

Lemma 4.1. Let $u_{1}, u_{2} \in U$, and $z \in Z$. Then

$$
u_{1}\left(u_{2} z\right)+u_{2}\left(u_{1} z\right)=\rho e+\left(u_{1} u_{2}\right) z,
$$

where $\rho=\varphi_{e}\left(u_{1}\left(u_{2} z\right)\right)+\varphi_{e}\left(u_{2}\left(u_{1} z\right)\right)$.

This follows immediately from linearization of Theorem 1.4(6). 
Proof. For the convenience of the reader we give the details. Using Theorem 1.4(6), write $u_{i}\left(u_{i} z\right)=\gamma_{i} e+\frac{1}{2} u_{i}^{2} z$, with $\gamma_{i} \in \mathbb{F}$, for $i \in\{1,2\}$. Then by Theorem 1.4 (6),

$$
\begin{aligned}
& \left(u_{1}+u_{2}\right)\left(\left(u_{1}+u_{2}\right) z\right)=\gamma e+\frac{1}{2}\left(u_{1}+u_{2}\right)^{2} z, \text { for some } \gamma \in \mathbb{F} \\
& \Longleftrightarrow u_{1}\left(u_{1} z\right)+u_{2}\left(u_{2} z\right)+u_{1}\left(u_{2} z\right)+u_{2}\left(u_{1} z\right) \\
& =\gamma e+\frac{1}{2} u_{1}^{2} z+\frac{1}{2} u_{2}^{2} z+\left(u_{1} u_{2}\right) z \\
& \Longleftrightarrow \gamma_{1} e+\frac{1}{2} u_{1}^{2} z+\gamma_{2} e+\frac{1}{2} u_{2}^{2} z+u_{1}\left(u_{2} z\right)+u_{2}\left(u_{1} z\right) \\
& =\gamma e+\frac{1}{2} u_{1}^{2} z+\frac{1}{2} u_{2}^{2} z+\left(u_{1} u_{2}\right) z \\
& \Longleftrightarrow\left(\gamma_{1}+\gamma_{2}\right) e+u_{1}\left(u_{2} z\right)+u_{2}\left(u_{1} z\right)=\gamma e+\left(u_{1} u_{2}\right) z \\
& \Longleftrightarrow u_{1}\left(u_{2} z\right)+u_{2}\left(u_{1} z\right)=\rho e+\left(u_{1} u_{2}\right) z .
\end{aligned}
$$

The lemma follows.

Lemma 4.2. Let $u \in U$ and $z_{1}, z_{2}, z \in Z$. Then

(1) $u\left(z_{1} z_{2}\right)=\left(u z_{1}\right) z_{2}+\left(u z_{2}\right) z_{1}$.

(2) $\left(u z^{2}\right) z=(u z) z^{2}=\frac{1}{2} u z^{3}$.

Proof. (1): By Theorem 1.4(2) we have

$$
\begin{aligned}
& 2\left(u\left(z_{1}+z_{2}\right)\right)\left(z_{1}+z_{2}\right)=u\left(z_{1}+z_{2}\right)^{2} \Longleftrightarrow \\
& 2\left(u z_{1}\right) z_{1}+2\left(u z_{2}\right) z_{2}+2\left[\left(u z_{1}\right) z_{2}+\left(u z_{2}\right) z_{1}\right]=u z_{1}^{2}+u z_{2}^{2}+2 u\left(z_{1} z_{2}\right) .
\end{aligned}
$$

Since $2\left(u z_{i}\right) z_{i}=u z_{i}^{2}$, for $i=1,2$, part (1) holds.

(2): Put $x=z, y=e$ and $v=u$ in identity (2.5) to conclude

$$
\begin{aligned}
0 & =\left[z, e u, z^{2}\right]+\left[e, u z, z^{2}\right]+\left[u, e z, z^{2}\right]+\left[z, z^{2}, e u\right] \\
& +\left[e, z^{2}, u z\right]+\left[u, z^{2}, z e\right]+2[x, z e, z u]+2[z, z u, z e],
\end{aligned}
$$

That is

$$
0=\frac{1}{2}(u z) z^{2}-\frac{1}{2}\left(u z^{2}\right) z+0+0+\frac{1}{2} u z^{3}-\frac{1}{2}\left(u z^{2}\right) z-\frac{1}{2}(u z) z^{2}+0+0+0 .
$$

Hence $\left(u z^{2}\right) z=\frac{1}{2} u z^{3}$. Also by (1), $u z^{3}=\left(u z^{2}\right) z+(u z) z^{2}$, so (2) holds.

Lemma 4.3. Let $u_{1}, u_{2} \in U$ and write $u_{1}^{2}=\delta_{1} e+z_{1}$ and $u_{2}^{2}=\delta_{2} e+z_{2}$, with $\delta_{i} \in \mathbb{F}$ and $z_{i} \in Z$, for $i=1,2$..

Let

$$
\begin{gathered}
u_{1} u_{2}=\delta_{12} e+z_{12} \text { and }\left(u_{1}+u_{2}\right)^{2}=\delta_{1+2} e+z_{1+2}, \\
\delta_{12}, \delta_{1+2} \in \mathbb{F}, z_{12}, z_{1+2} \in Z .
\end{gathered}
$$

Then

(1) $\delta_{1+2}=\delta_{1}+\delta_{2}+2 \delta_{12}$.

(2)

$$
\begin{gathered}
\delta_{1+2}\left(u_{1}+u_{2}\right)= \\
\delta_{1} u_{1}+\delta_{2} u_{2}+u_{1}^{2} u_{2}+u_{2}^{2} u_{1}+\delta_{12} u_{1}+\delta_{12} u_{2}+2 z_{12} u_{1}+2 z_{12} u_{2} .
\end{gathered}
$$


(3) $u_{1}^{2} u_{2}+u_{2}^{2} u_{1}-\delta_{12} u_{1}-\delta_{2} u_{1}-\delta_{12} u_{2}-\delta_{1} u_{2}+2 z_{12}\left(u_{1}+u_{2}\right)=0$.

(4) $u_{1}^{2} u_{2}-\delta_{12} u_{1}-\delta_{1} u_{2}+2 u_{1} z_{12}=0$.

(5) $2 u_{1}\left(u_{1} u_{2}\right)=-u_{1}^{2} u_{2}+\delta_{1} u_{2}+2 \delta_{12} u_{1}$.

(6) $u_{2} z_{1}-\delta_{12} u_{1}-\frac{1}{2} \delta_{1} u_{2}+2 u_{1} z_{12}=0$.

(7) $2\left(u_{1} z_{12}\right) z_{1}=\frac{1}{2} \delta_{12} \delta_{1} u_{1}$.

(8) $2\left(u_{1}\left(u_{1} u_{2}\right)\right) z_{1}=\delta_{12} \delta_{1} u_{1}$.

Proof. (1): We have

$$
\begin{aligned}
\delta_{1+2} e+z_{1+2} & =\left(u_{1}+u_{2}\right)^{2}=u_{1}^{2}+u_{2}^{2}+2 u_{1} u_{2} \\
& =\left(\delta_{1}+\delta_{2}+2 \delta_{12}\right) e+z_{1}+z_{2}+2 z_{12} .
\end{aligned}
$$

(2): We have

$$
\begin{aligned}
\delta_{1+2}\left(u_{1}+u_{2}\right) & =\left(u_{1}+u_{2}\right)^{3}=u_{1}^{3}+u_{2}^{3}+u_{1}^{2} u_{2}+u_{2}^{2} u_{1}+2\left(u_{1} u_{2}\right) u_{1}+2\left(u_{1} u_{2}\right) u_{2} \\
& =\delta_{1} u_{1}+\delta_{2} u_{2}+u_{1}^{2} u_{2}+u_{2}^{2} u_{1}+\delta_{12} u_{1}+2 z_{12} u_{1}+\delta_{12} u_{2}+2 z_{12} u_{2} .
\end{aligned}
$$

(3): Just replace $\delta_{1+2}$ by $\delta_{1}+\delta_{2}+2 \delta_{12}$ in (2).

(4): Replace $u_{1}$ with $\alpha u_{1}$ in (3), where $0 \neq \alpha \in \mathbb{F}$. Then $\delta_{1}$ should be replaced with $\alpha^{2} \delta_{1}, \delta_{12}$ with $\alpha \delta_{12}$ and $z_{12}$ with $\alpha z_{12}$. We get from (3),

$\alpha^{2} u_{1}^{2} u_{2}+\alpha u_{2}^{2} u_{1}-\alpha^{2} \delta_{12} u_{1}-\alpha \delta_{2} u_{1}-\alpha \delta_{12} u_{2}-\alpha^{2} \delta_{1} u_{2}+2 \alpha z_{12}\left(\alpha u_{1}+u_{2}\right)=0$.

dividing by $\alpha$ we see that

(*) $\alpha u_{1}^{2} u_{2}+u_{2}^{2} u_{1}-\alpha \delta_{12} u_{1}-\delta_{2} u_{1}-\delta_{12} u_{2}-\alpha \delta_{1} u_{2}+2 z_{12}\left(\alpha u_{1}+u_{2}\right)=0$.

Since $\alpha$ is arbitrary we get (4).

(5): Replacing in (4) $z_{12}=u_{1} u_{2}-\delta_{12} e$ we get

$$
\begin{gathered}
u_{1}^{2} u_{2}-\delta_{12} u_{1}-\delta_{1} u_{2}+2\left(u_{1} u_{2}-\delta_{12} e\right) u_{1}=0 \\
\Longleftrightarrow 2 u_{1}\left(u_{1} u_{2}\right)=-u_{1}^{2} u_{2}+\delta_{1} u_{2}+2 \delta_{12} u_{1} .
\end{gathered}
$$

(6): This is obtained from (4) by replacing $u_{1}^{2}$ with $\delta_{1} e+z_{1}$.

(7): Now multiply (6) by $z_{1}$. We get

$$
\begin{aligned}
& \left(u_{2} z_{1}\right) z_{1}-\delta_{12} u_{1} z_{1}-\frac{1}{2} \delta_{1} u_{2} z_{1}+2\left(u_{1} z_{12}\right) z_{1}=0 \\
& \stackrel{(i)}{\Longleftrightarrow} \frac{1}{2} u_{2} z_{1}^{2}-\frac{1}{2} \delta_{1} \delta_{12} u_{1}-\frac{1}{2} \delta_{1} u_{2} z_{1}+2\left(u_{1} z_{12}\right) z_{1}=0 \\
& \stackrel{(i i)}{\Longleftrightarrow} \frac{1}{2} \delta_{1} u_{2} z_{1}-\frac{1}{2} \delta_{1} \delta_{12} u_{1}-\frac{1}{2} \delta_{1} u_{2} z_{1}+2\left(u_{1} z_{12}\right) z_{1}=0 \\
& \Longleftrightarrow-\frac{1}{2} \delta_{1} \delta_{12} u_{1}+2\left(u_{1} z_{12}\right) z_{1}=0 .
\end{aligned}
$$

Here (i) holds because by Lemma 3.1, $u_{1} z_{1}=\frac{1}{2} \delta_{1} u_{1}$, and (ii) holds by Lemma 3.1 . 
(8): Replacing $z_{12}$ by $u_{1} u_{2}-\delta_{12} e$ in (7), we get

$$
\begin{aligned}
& 2\left(u_{1} z_{12}\right) z_{1}=\frac{1}{2} \delta_{12} \delta_{1} u_{1} \\
& \Longleftrightarrow 2\left(u_{1}\left(u_{1} u_{2}-\delta_{12} e\right)\right) z_{1}=\frac{1}{2} \delta_{12} \delta_{1} u_{1} \\
& \Longleftrightarrow 2\left(u_{1}\left(u_{1} u_{2}\right)\right) z_{1}-\delta_{12} u_{1} z_{1}=\frac{1}{2} \delta_{12} \delta_{1} u_{1} \\
& \quad \Longleftrightarrow 2\left(u_{1}\left(u_{1} u_{2}\right)\right) z_{1}-\frac{1}{2} \delta_{12} \delta_{1} u_{1}=\frac{1}{2} \delta_{12} \delta_{1} u_{1} \\
& \quad \Longleftrightarrow 2\left(u_{1}\left(u_{1} u_{2}\right)\right) z_{1}=\delta_{12} \delta_{1} u_{1} .
\end{aligned}
$$

Where (i) holds by Lemma 3.1 .

Lemma 4.4. Let $u_{1}, u_{2}, u_{3} \in U$. Then

(1) $u_{1}\left(u_{2} u_{3}\right)+\left(u_{1} u_{2}\right) u_{3}+\left(u_{1} u_{3}\right) u_{2}=\delta_{u_{2} u_{3}} u_{1}+\delta_{u_{1} u_{3}} u_{2}+\delta_{u_{1} u_{2}} u_{3}$.

(2) $u_{1}^{2} u_{2}+2 u_{1}\left(u_{1} u_{2}\right)=\delta_{u_{1}^{2}} u_{2}+2 \delta_{u_{1} u_{2}} u_{1}$.

(3) $u_{1}^{2}\left(u_{2} z\right)+2 u_{1}\left(u_{1}\left(u_{2} z\right)\right)=\delta_{u_{1}^{2}} u_{2} z+2 \delta_{u_{1}\left(u_{2} z\right)} u_{1}$.

(4) $\left(u_{1}^{2} u_{2}\right) z=-2 u_{1}\left(\left(u_{1} u_{2}\right) z\right)+2\left(u_{1} u_{2}\right)\left(u_{1} z\right)+\delta_{u_{1}^{2}} u_{2} z$.

(5) $2 u_{1}^{2}\left(u_{1} z\right)=\delta_{u_{1}^{2}} u_{1} z+\delta_{u_{1}\left(u_{1} z\right)} u_{1}$.

Proof. (1): Put $u_{2}+u_{3}$ in place of $u_{1}$, and $u_{1}$ in place of $u_{2}$ in Lemma 4.3(5) to get:

$$
\begin{aligned}
& 2\left(u_{1}\left(u_{2}+u_{3}\right)\right)\left(u_{2}+u_{3}\right)= \\
& -u_{1}\left(u_{2}+u_{3}\right)^{2}+\delta_{\left(u_{2}+u_{3}\right)^{2}} u_{1}+2 \delta_{u_{1} u_{2}}\left(u_{2}+u_{3}\right)+2 \delta_{u_{1} u_{3}}\left(u_{2}+u_{3}\right) \\
& \Longleftrightarrow \\
& 2 u_{2}\left(u_{2} u_{1}\right)+2 u_{3}\left(u_{3} u_{1}\right)+2\left(u_{1} u_{2}\right) u_{3}+2\left(u_{1} u_{3}\right) u_{2} \\
& =-u_{2}^{2} u_{1}-u_{3}^{2} u_{1}-2 u_{1}\left(u_{2} u_{3}\right)+\delta_{u_{2}^{2}} u_{1}+\delta_{u_{3}^{2}} u_{1}+2 \delta_{u_{2} u_{3}} u_{1} \\
& +2 \delta_{u_{1} u_{2}} u_{2}+2 \delta_{u_{1} u_{2}} u_{3}+2 \delta_{u_{1} u_{3}} u_{3}+2 \delta_{u_{1} u_{3}} u_{2} \\
& \stackrel{(i)}{\Longleftrightarrow} \\
& 2\left(u_{1} u_{2}\right) u_{3}+2\left(u_{1} u_{3}\right) u_{2}=-2 u_{1}\left(u_{2} u_{3}\right)+2 \delta_{u_{2} u_{3}} u_{1}+2 \delta_{u_{1} u_{3}} u_{2}+2 \delta_{u_{1} u_{2}} u_{3} \\
& \Longleftrightarrow \\
& u_{2}\left(u_{3} u_{1}\right)+u_{3}\left(u_{2} u_{1}\right)+\left(u_{2} u_{3}\right) u_{1}=\delta_{u_{2} u_{3}} u_{1}+\delta_{u_{1} u_{3}} u_{2}+\delta_{u_{1} u_{2}} u_{3} .
\end{aligned}
$$

Where $(i)$ is obtained by applying Lemma 4.3(5) twice.

(2): This is obtained by replacing $u_{2}$ with $u_{1}$ and $u_{3}$ with $u_{2}$ in (1)

(3): Put $u_{2} z$ in place of $u_{2}$ in (2). 
(4): Multiplying (2) by $z$ we have

$$
\begin{aligned}
& \left(u_{1}^{2} u_{2}\right) z=-2\left(u_{1}\left(u_{1} u_{2}\right)\right) z+\delta_{u_{1}^{2}} u_{2} z+2 \delta_{u_{1} u_{2}} u_{1} z \\
& =-2\left(u_{1}\left(\delta_{u_{1} u_{2}} e+z_{u_{1} u_{2}}\right)\right) z+\delta_{u_{1}^{2}} u_{2} z+2 \delta_{u_{1} u_{2}} u_{1} z \\
& =-\delta_{u_{1} u_{2}} u_{1} z-2\left(u_{1} z_{u_{1} u_{2}}\right) z+\delta_{u_{1}^{2}} u_{2} z+2 \delta_{u_{1} u_{2}} u_{1} z \\
& \stackrel{(i)}{=}-2 u_{1}\left(z_{u_{1} u_{2}} z\right)+2\left(u_{1} z\right) z_{u_{1} u_{2}}+\delta_{u_{1}^{2}} u_{2} z+\delta_{u_{1} u_{2}} u_{1} z \\
& =-2 u_{1}\left(\left(u_{1} u_{2}\right) z\right)+2\left(u_{1} z\right)\left(u_{1} u_{2}-\delta_{u_{1} u_{2}} e\right)+\delta_{u_{1}^{2}} u_{2} z+\delta_{u_{1} u_{2}} u_{1} z \\
& =-2 u_{1}\left(\left(u_{1} u_{2}\right) z\right)+2\left(u_{1} z\right)\left(u_{1} u_{2}\right)+\delta_{u_{1}^{2}} u_{2} z .
\end{aligned}
$$

Where $(i)$ is obtained by using Lemma $4.2(1)$.

(5): Put $u_{1}$ in place of $u_{2}$ in (3). We get

$$
\begin{aligned}
& u_{1}^{2}\left(u_{1} z\right)+2 u_{1}\left(u_{1}\left(u_{1} z\right)\right)=\delta_{u_{1}^{2}} u_{1} z+2 \delta_{u_{1}\left(u_{1} z\right)} u_{1} \\
& \stackrel{(i)}{\Longleftrightarrow} u_{1}^{2}\left(u_{1} z\right)+2 u_{1}\left(\delta_{u_{1}\left(u_{1} z\right)} e+\frac{1}{2} u_{1}^{2} z\right)=\delta_{u_{1}^{2}} u_{1} z+2 \delta_{u_{1}\left(u_{1} z\right)} u_{1} \\
& \Longleftrightarrow u_{1}^{2}\left(u_{1} z\right)+\delta_{u_{1}\left(u_{1} z\right)} u_{1}+u_{1}\left(u_{1}^{2} z\right)=\delta_{u_{1}^{2}} u_{1} z+2 \delta_{u_{1}\left(u_{1} z\right)} u_{1} \\
& \quad \Longleftrightarrow 2 u_{1}^{2}\left(u_{1} z\right)=\delta_{u_{1}^{2}} u_{1} z+\delta_{u_{1}\left(u_{1} z\right)} u_{1} .
\end{aligned}
$$

Here $(i)$ holds by Theorem 1.4(6), and (ii) is by Lemma 6.1 below. (Indeed Lemma 6.1 naturally belongs in $\S 6$.)

Lemma 4.5. For $u, u_{1}, u_{2} \in U, z, z_{1}, z_{2} \in Z$ we have

(1) $2(u z)^{2}+u^{2} z^{2}=u\left(u z^{2}\right)+\left(u^{2} z\right) z$.

(2) $4\left(u_{1} z\right)\left(u_{2} z\right)+2\left(u_{1} u_{2}\right) z^{2}=u_{1}\left(u_{2} z^{2}\right)+u_{2}\left(u_{1} z^{2}\right)+2\left(\left(u_{1} u_{2}\right) z\right) z$.

(3) $4\left(u z_{1}\right)\left(u z_{2}\right)+2 u^{2}\left(z_{1} z_{2}\right)=2 u\left(u\left(z_{1} z_{2}\right)\right)+\left(u^{2} z_{1}\right) z_{2}+\left(u^{2} z_{2}\right) z_{1}$.

(4) $2(u z)^{2} z+\frac{1}{2} u^{2} z^{2}=\left(\left(u^{2} z\right) z\right) z$.

(5) $\delta_{u\left(u z^{2}\right)}=2 \delta_{(u z)^{2}}$.

(6) $4 \delta_{\left(u_{1} z\right)\left(u_{2} z\right)}=\delta_{u_{1}\left(u_{2} z^{2}\right)+u_{2}\left(u_{1} z^{2}\right)}$.

(7) $2 \delta_{\left(u z_{1}\right)\left(u z_{2}\right)}=\delta_{u\left(u\left(z_{1} z_{2}\right)\right)}$.

Proof. Part (1) follows from Theorem 1.4(4) using Theorem 1.4(2\&6). For part (2) replace $u$ by $u_{1}+u_{2}$ is (1), and then use (1) twice, to get:

$$
\begin{aligned}
& 2\left(\left(u_{1}+u_{2}\right) z\right)^{2}+\left(u_{1}+u_{2}\right)^{2} z^{2}=\left(u_{1}+u_{2}\right)\left(\left(u_{1}+u_{2}\right) z^{2}\right)+\left(\left(u_{1}+u_{2}\right)^{2} z\right) z \\
& \Longleftrightarrow 2\left(u_{1} z\right)^{2}+2\left(u_{2} z\right)^{2}+4\left(u_{1} z\right)\left(u_{2} z\right)+u_{1}^{2} z^{2}+u_{2}^{2} z^{2}+2\left(u_{1} u_{2}\right) z^{2} \\
& =u_{1}\left(u_{1} z^{2}\right)+u_{2}\left(u_{2} z^{2}\right)+u_{1}\left(u_{2} z^{2}\right)+u_{2}\left(u_{1} z^{2}\right)+\left(u_{1}^{2} z\right) z+\left(u_{2}^{2} z\right) z+2\left(\left(u_{1} u_{2}\right) z\right) z \\
& \Longleftrightarrow 4\left(u_{1} z\right)\left(u_{2} z\right)+2\left(u_{1} u_{2}\right) z^{2}=u_{1}\left(u_{2} z^{2}\right)+u_{2}\left(u_{1} z^{2}\right)+2\left(\left(u_{1} u_{2}\right) z\right) z .
\end{aligned}
$$

This shows (2).

(3): Replacing $z$ with $z_{1}+z_{2}$ in (1) we get

$$
2\left(u\left(z_{1}+z_{2}\right)\right)^{2}+u^{2}\left(z_{1}+z_{2}\right)^{2}=u\left(u\left(z_{1}+z_{2}\right)^{2}\right)+\left(u^{2}\left(z_{1}+z_{2}\right)\right)\left(z_{1}+z_{2}\right) .
$$




$$
\begin{aligned}
& \text { Or } \\
& 2\left(u z_{1}\right)^{2}+2\left(u z_{2}\right)^{2}+4\left(u z_{1}\right)\left(u z_{2}\right)+u^{2} z_{1}^{2}+u^{2} z_{2}^{2}+2 u^{2}\left(z_{1} z_{2}\right) \\
& =u\left(u z_{1}^{2}\right)+u\left(u z_{2}^{2}\right)+2 u\left(u\left(z_{1} z_{2}\right)\right)+\left(u^{2} z_{1}\right) z_{1}+\left(u^{2} z_{2}\right) z_{2}+\left(u^{2} z_{1}\right) z_{2}+\left(u^{2} z_{2}\right) z_{1} .
\end{aligned}
$$

So using (1) we get (3).

(4): Multiply (1) by $z$ and note that $\left(u\left(u z^{2}\right)\right) z=\frac{1}{2} u^{2} z^{2}$, by Theorem 1.4(6).

Parts (5), (6) and (7) are consequences of the previous parts.

\section{Half-aXes in $A$}

Let $e \neq f \in A$ be another half-axis in $A$ and write

$$
f=\gamma e+u_{1}+z \quad \gamma \in \mathbb{F}, u_{1} \in U, z \in Z .
$$

Thus the subalgebra $A_{e, f}$ of $A$ generated by $e$ and $f$ is a primitive axial algebra of Jordan type half. We use [HSS1] to deduce information on $A_{e, f}$. We use the notation of [HSS1. Let

$$
\sigma:=\sigma_{e, f}, \pi:=\pi_{e, f}, \delta_{1}:=\delta_{u_{1}^{2}} \text { and } z_{1}:=z_{u_{1}^{2}} .
$$

Lemma 5.1. We have

(1) $u_{1} z=-\frac{1}{2}(\gamma-1) u_{1}$;

(2) $z_{1}=z-z^{2}$

(3) $\gamma-\gamma^{2}=\delta_{1}$.

Proof. We have

$$
f^{2}=\gamma^{2} e+u_{1}^{2}+z^{2}+\gamma u_{1}+2 u_{1} z=\left(\gamma^{2}+\delta_{1}\right) e+\left(\gamma u_{1}+2 u_{1} z\right)+\left(z_{1}+z^{2}\right) .
$$

Since $f^{2}=f=\gamma e+u_{1}+z$, the lemma follows.

Proposition 5.2. We have

(1) $\sigma=\frac{\gamma-1}{2} e-\frac{1}{2} z$

(2) $\pi=\frac{\gamma-1}{2}$.

(3) $z^{2}=(1-\gamma) z$.

(4) $z_{1}=\gamma z$, in particular

(5) if $\gamma \neq 0$, then $u_{1} \neq 0$, and $z=\frac{u_{1}^{2}}{\gamma}-\frac{\delta_{1}}{\gamma} e$.

(6) If $\gamma=0$, then $f=u_{1}+z$, with $u_{1}^{2}=0$ and $z u_{1}=\frac{1}{2} u_{1}$. In particular $u_{1} \in A_{1 / 2}(f)$.

Proof. (1): We have ef $=\gamma e+\frac{1}{2} u_{1}$, hence

$$
\begin{aligned}
\sigma=e f-\frac{1}{2} e-\frac{1}{2} f & =\gamma e+\frac{1}{2} u_{1}-\frac{1}{2} e-\frac{1}{2} \gamma e-\frac{1}{2} u_{1}-\frac{1}{2} z \\
& =\frac{1}{2}(\gamma-1) e-\frac{1}{2} z .
\end{aligned}
$$

(2): $\quad \sigma e=\pi e \Longrightarrow \frac{1}{2}(\gamma-1) e=\pi e$, so (2) holds. 
(3): Note that by (2), $\sigma=\pi e-\frac{1}{2} z$, hence

$$
\sigma^{2}=\pi \sigma \Longrightarrow \pi^{2} e+\frac{1}{4} z^{2}=\pi^{2} e-\frac{1}{2} \pi z
$$

Hence $\frac{1}{4} z^{2}=-\frac{1}{2} \pi z$. It follows that $z^{2}=-2 \pi z=(1-\gamma) z$.

(4): By Lemma 5.1(2) and by (3), we get $z_{1}=z-z^{2}=z+(\gamma-1) z=\gamma z$.

(5): If $u_{1}=0$, then by Lemma $5.1(2 \& 3), \gamma=1$ and $z^{2}=z$. But then $f$ is not a half-axis, since $f e=e$. Now (5) follows from (4) since $z_{1}=u_{1}^{2}-\delta_{1} e$.

(6): Suppose $\gamma=0$. Then, by Lemma 5.1(3), $\delta_{1}=0$. Also by (4), $z_{1}=0$, so $u_{1}^{2}=0$. Also, by [5.1(1), $u_{1} z=\frac{1}{2} u_{1}$. Thus $f u_{1}=z u_{1}=\frac{1}{2} u_{1}$, and $u_{1} \in A_{1 / 2}(f)$.

\section{Special CASEs of the Jordan identity}

Throughout this section we assume that the identities $x^{2} x^{2}=x x^{3}$ and $x^{3} x^{2}=x x^{4}$ hold strictly in $A$. In this section we deduce certain identities that are specific cases of the general Jordan identity. These indicate that $A$ "tends" to be a Jordan algebra.

Lemma 6.1. Let $u_{1} \in U$ and $z \in Z$, then $u_{1}^{2}\left(u_{1} z\right)=u_{1}\left(u_{1}^{2} z\right)$.

Proof. By Lemma 3.1 we have

$$
u_{1}^{2}\left(u_{1} z\right)=\left(\delta_{u_{1}^{2}} e+z_{u_{1}^{2}}\right) u_{1} z=\frac{1}{2} \delta_{u_{1}^{2}} u_{1} z+\left(u_{1} z\right) z_{u_{1}^{2}}=\left(u_{1} z_{u_{1}^{2}}\right) z+\left(u_{1} z\right) z_{u_{1}^{2}} .
$$

But also $u_{1}\left(u_{1}^{2} z\right)=u_{1}\left(z_{u_{1}^{2}} z\right)$. So the lemma follows from Lemma 4.2(1).

Lemma 6.2. Let $u_{1}, u_{2} \in U$ and write $u_{1}^{2}=\delta_{1} e+z_{1}$. Assume that $u_{1}^{2} \notin Z$. Then

$$
\delta_{u_{1}\left(u_{2} z_{1}\right)}=\frac{1}{2} \delta_{1} \delta_{u_{1} u_{2}} .
$$

Proof. Recall from Lemma 3.1 that $z_{1}^{2}=\delta_{1} z_{1}$ and $u_{1} z_{1}=\frac{1}{2} \delta_{1} u_{1}$. Hence also $u_{1} z_{1}^{2}=\frac{1}{2} \delta_{1}^{2} u_{1}$. Thus, by Lemma 4.5(6), with $z=z_{1}$ we have

$$
\begin{aligned}
& 4 \delta_{\left(u_{1} z_{1}\right)\left(u_{2} z_{1}\right)}=\delta_{u_{1}\left(u_{2} z_{1}^{2}\right)}+\delta_{u_{2}\left(u_{1} z_{1}^{2}\right)} \\
& \Longleftrightarrow 2 \delta_{1} \delta_{u_{1}\left(u_{2} z_{1}\right)}=\delta_{1} \delta_{u_{1}\left(u_{2} z_{1}\right)}+\frac{1}{2} \delta_{1}^{2} \delta_{u_{1} u_{2}} \\
& \Longleftrightarrow 2 \delta_{u_{1}\left(u_{2} z_{1}\right)}=\delta_{u_{1}\left(u_{2} z_{1}\right)}+\frac{1}{2} \delta_{1} \delta_{u_{1} u_{2}} .
\end{aligned}
$$

Lemma 6.3. Let $u_{1} \in U$ such that $u_{1}^{2} \notin Z$. Then $u_{1}^{2}\left(u_{1} u_{2}\right)=u_{1}\left(u_{1}^{2} u_{2}\right)$.

Proof. Write $u_{1}^{2}=\delta_{1} e+z_{1}$. We have

$$
u_{1}^{2}\left(u_{1} u_{2}\right)=\left(\delta_{1} e+z_{1}\right)\left(u_{1} u_{2}\right)=\delta_{1} e\left(u_{1} u_{2}\right)+\left(u_{1} u_{2}\right) z_{1},
$$

and using Lemma 3.1,

$$
\begin{gathered}
u_{1}\left(u_{1}^{2} u_{2}\right)=u_{1}\left(\left(\delta_{1} e+z_{1}\right) u_{2}\right)=\frac{1}{2} \delta_{1}\left(u_{1} u_{2}\right)+u_{1}\left(u_{2} z_{1}\right) \\
=u_{2}\left(u_{1} z_{1}\right)+u_{1}\left(u_{2} z_{1}\right) .
\end{gathered}
$$


Hence we need to show that

$$
\delta_{1} e\left(u_{1} u_{2}\right)+\left(u_{1} u_{2}\right) z_{1}=u_{2}\left(u_{1} z_{1}\right)+u_{1}\left(u_{2} z_{1}\right) .
$$

By Lemma 4.1,

$$
\left(u_{1} u_{2}\right) z_{1}=u_{1}\left(u_{2} z_{1}\right)+u_{2}\left(u_{1} z_{1}\right)-e\left(u_{1}\left(u_{2} z_{1}\right)+u_{2}\left(u_{1} z_{1}\right)\right),
$$

so we must show that

$$
\delta_{1} e\left(u_{1} u_{2}\right)=e\left(u_{2}\left(u_{1} z_{1}\right)+u_{1}\left(u_{2} z_{1}\right)\right)=\frac{1}{2} \delta_{1} e\left(u_{1} u_{2}\right)+e\left(u_{1}\left(u_{2} z_{1}\right)\right) .
$$

Or

$$
\frac{1}{2} \delta_{1} e\left(u_{1} u_{2}\right)=e\left(u_{1}\left(u_{2} z_{1}\right)\right) .
$$

But this was shown in Lemma 6.2,

Lemma 6.4. Let $u \in U$ and $z \in Z$, then $\left(u^{2} z^{2}\right) z=\left(u^{2} z\right) z^{2}$.

Proof. By Lemma 4.5(4), we have

$$
2(u z)^{2} z+\frac{1}{2}\left(u^{2} z^{2}\right) z=\left(\left(u^{2} z\right) z\right) z .
$$

Next, by Lemma 4.5(2), putting $u$ in place of $u_{1}$ and $u z$ in place of $u_{2}$ we get.

$$
4(u z)((u z) z)+2(u(u z)) z^{2}=u\left((u z) z^{2}\right)+(u z)\left(u z^{2}\right)+2((u(u z)) z) z .
$$

Using Theorem 1.4(2\&6) and Lemma 4.2(2) we get

$$
(u z)\left(u z^{2}\right)+\left(u^{2} z\right) z^{2}=\frac{1}{2} u\left(u z^{3}\right)+\left(\left(u^{2} z\right) z\right) z .
$$

Note that the $Z$-part of $(u z)\left(u z^{2}\right)=2(u z)((u z) z)$ is $(u z)^{2} z$, using Theorem 1.4 (2\&6). Comparing the $Z$-parts in equation (6.2) (using again 1.4(6)) we see that

$$
(u z)^{2} z+\left(u^{2} z\right) z^{2}=\frac{1}{4} u^{2} z^{3}+\left(\left(u^{2} z\right) z\right) z .
$$

Note now that by Lemma 4.1 and Lemma $4.2(2),(u(u z)) z^{2}$ equals the $Z$-part of

$(u z)\left(u z^{2}\right)+u\left((u z) z^{2}\right)=2(u z)((u z) z)+u\left((u z) z^{2}\right)=2(u z)((u z) z)+\frac{1}{2} u\left(u z^{3}\right)$.

Using Theorem 1.4(6) we get that

$$
\frac{1}{2}\left(u^{2} z\right) z^{2}=(u z)^{2} z+\frac{1}{4} u^{2} z^{3} .
$$

or

$$
\frac{1}{4} u^{2} z^{3}=\frac{1}{2}\left(u^{2} z\right) z^{2}-(u z)^{2} z .
$$

Inserting equation (6.4) in equation (6.3) we get

$$
2(u z)^{2} z+\frac{1}{2}\left(u^{2} z\right) z^{2}=\left(\left(u^{2} z\right) z\right) z .
$$

Comparing equations (6.1) and (6.5) we get the lemma. 


\section{IDENTITIES BETWEEN EIGENSPACES OF $\operatorname{ad}_{e}$, AND THE JORDAN IDENTITY}

The purpose of this section is to prove the following Theorem.

Theorem 7.1. Let $A$ be a commutative non-associative algebra over $\mathbb{F}$ and suppose $e \in A$ is a half-axis of $A$. Then $A$ is a Jordan algebra if and only if the following identities hold, for all elements $u, v \in A_{1 / 2}(e), z, z^{\prime} \in A_{0}(e)$.

(1) $u\left(u^{2} e\right)=\frac{1}{2} u^{3}$.

(2) $u\left(z z^{\prime}\right)=(u z) z^{\prime}+\left(u z^{\prime}\right) z$.

(3) $u\left(u^{2} z\right)=u^{2}(u z)$.

(4) $u^{2}\left(z z^{\prime}\right)+2(u z)\left(u z^{\prime}\right)=\left(u^{2} z^{\prime}\right) z+2 u\left((u z) z^{\prime}\right)$.

(5) $\left(u z^{\prime}\right) z^{2}+2(u z)\left(z z^{\prime}\right)=u\left(z^{2} z^{\prime}\right)+2\left((u z) z^{\prime}\right) z$.

(6) $z\left(z^{2} z^{\prime}\right)=z^{2}\left(z z^{\prime}\right)$

(7) $2((u z) v) e+(u v) z=u(v z)+v(u z)$.

(8) $u\left(u^{2} v\right)=u^{2}(u v)$.

(9) $2 u(v(u z))+\left(u^{2} v\right) z=2(u v)(u z)+u^{2}(v z)$.

(10) $2((u z) v) z+u\left(v z^{2}\right)=2(u z)(v z)+(u v) z^{2}$.

(11) $z\left(z^{2} v\right)=z^{2}(z v)$.

Proof. As noted in the introduction, since tensor products commute with direct sums, $e$ is a half-axis in $A$ if and only if it is a half axis in $A_{\mathbb{K}}$, for any field extension $\mathbb{K} / \mathbb{F}$.

Let $r, s, t$ be independent variables and $\mathbb{K}:=\mathbb{F}(r, s, t)$ the corresponding rational function field. We canonically identify $A \subseteq A_{\mathbb{K}}$ as an $\mathbb{F}$-subalgebra and, for arbitrary elements $u \in U:=A_{1 / 2}(e), z \in Z:=A_{0}(e)$, consider the quantity

$$
x:=r e+s u+t z \in A_{\mathbb{K}} .
$$

Note that the fusion rules imply

$$
x^{2}=r^{2} e+r s u+s^{2} u^{2}+2 s t u z+t^{2} z^{2} .
$$

Since Jordan algebras are stable under base change, A is a Jordan algebra if and only if $A_{\mathbb{K}}$ is a Jordan algebra. Hence $A$ is a Jordan algebra if and only if for all $u, v \in U, z, z^{\prime} \in Z$ and $x$ as above, the relations

$$
\begin{aligned}
x\left(x^{2} e\right) & =x^{2}(x e) . \\
x\left(x^{2} z^{\prime}\right) & =x^{2}\left(x z^{\prime}\right) . \\
x\left(x^{2} v\right) & =x^{2}(x v) .
\end{aligned}
$$

hold. Expanding both sides of equations (7.1)-(7.3), viewing the result as polynomials in $r, s, t$, over $A$, and comparing coefficients, straightforward computations, which are only mildly tedious and are left to the reader, show that equations (7.1)-(7.3) are equivalent to (1)-(11) of the theorem.

As a corollary we get the following theorem 
Theorem 7.2. Let $A$ be a commutative non-associative algebra over $\mathbb{F}, e \in$ $A$ a half-axis, and suppose the identities $x^{2} x^{2}=x x^{3}, x^{3} x^{2}=x x^{4}$ hold strictly in $A$. Then $A$ is a Jordan algebra if and only if the following identities hold for all $u_{1}, u_{2} \in U$ and $z_{1}, z_{2} \in Z$ :

(i) $u_{1}\left(u_{1}^{2} u_{2}\right)=u_{1}^{2}\left(u_{1} u_{2}\right)$.

(ii) $\left(u_{1}^{2} u_{2}\right) z_{1}+2 u_{1}\left(u_{2}\left(u_{1} z_{1}\right)\right)=u_{1}^{2}\left(u_{2} z_{1}\right)+\left(u_{1} u_{2}\right)\left(u_{1} z_{1}\right)$.

(iii) $\left(u_{1}^{2} z_{2}\right) z_{1}+2 u_{1}\left(\left(u_{1} z_{1}\right) z_{2}\right)=u_{1}^{2}\left(z_{1} z_{2}\right)+2\left(u_{1} z_{1}\right)\left(u_{1} z_{2}\right)$.

(iv) $u_{1}\left(u_{2} z_{1}^{2}\right)+2\left(u_{2}\left(u_{1} z_{1}\right)\right) z_{1}=\left(u_{1} u_{2}\right) z_{1}^{2}+2\left(u_{1} z_{1}\right)\left(u_{2} z_{1}\right)$.

(v) $z_{1}\left(z_{1}^{2} z_{2}\right)=z_{1}^{2}\left(z_{1} z_{2}\right)$.

(vi) $\left(u_{1} u_{2}\right) z_{1}+2 e\left(u_{2}\left(u_{1} z_{1}\right)\right)=u_{1}\left(u_{2} z_{1}\right)+u_{2}\left(u_{1} z_{1}\right)$.

Proof. After a change of notation, the identities $(i)-(v i)$ agree with identities (8), (9), (4), (10), (6), (7) of Theorem 7.1. Hence Theorem 7.1 shows that, if $A$ is a Jordan algebra, then $(i)-(v i)$ hold.

Conversely, suppose $(i)-(v i)$ hold. We must prove identities (1), (2), (3), (5) and (11) of Theorem 7.1. We use Theorem 1.4.

Identity (1) of Theorem 7.1 follows from Theorem 1.4(1) and Lemma 3.1. while identity (2) is Lemma 4.2(1), and identity (3) is Lemma 6.1. Also identity (11) is Lemma $4.2(2)$.

Finally we prove identity (5) of Theorem 7.1 . We claim that

$$
2(u z)\left(z z^{\prime}\right)=\left(u z^{2}\right) z^{\prime}+2\left((u z) z^{\prime}\right) z .
$$

Indeed using Theorem $\mathbf{7 . 1}(2)$ twice we get

$$
2(u z)\left(z z^{\prime}\right)=2((u z) z) z^{\prime}+2\left((u z) z^{\prime}\right) z=\left(u z^{2}\right) z^{\prime}+2\left((u z) z^{\prime}\right) z .
$$

Combining identities (7.4) with Theorem [7.1(2), we obtain

$\left(u z^{\prime}\right) z^{2}+2(u z)\left(z z^{\prime}\right)=\left(u z^{\prime}\right) z^{2}+\left(u z^{2}\right) z^{\prime}+2\left((u z) z^{\prime}\right) z=u\left(z^{2} z^{\prime}\right)+2\left((u z) z^{\prime}\right) z$, as desired.

\section{A CONDition For $A$ TO BE A Jordan ALGEBRA}

Throughout this section we assume that the identities $x^{2} x^{2}=x x^{3}$ and $x^{3} x^{2}=x x^{4}$ hold strictly in $A$. We consider the condition

$(*) \quad \varphi_{e}\left(u_{1}\left(u_{2} z\right)\right)=\varphi_{e}\left(u_{2}\left(u_{1} z\right)\right)$, for all $u_{1}, u_{2} \in U$ and $z \in Z$.

In this section we will prove:

Theorem 8.1. Assume that (*) holds. Then identities (i)-(iv) and identity (vi) of Theorem 7.2 hold, for all $u_{1}, u_{2} \in U$ and $z_{1}, z_{2} \in Z$.

Using Theorem 8.1 we easily deduce:

Theorem 8.2. Assume that $(*)$ holds. Then $x\left(x^{2} u\right)=x^{2}(x u)$, for all $x \in A$ and $u \in U$.

Theorem 8.3. Assume that $(*)$ holds and let $z \in Z$. Then the following are equivalent 
(i) $x\left(x^{2} z\right)=x^{2}(x z)$, for all $x \in A$.

(ii) $z_{1}\left(z_{1}^{2} z\right)=z_{1}^{2}\left(z_{1} z\right)$, for all $z_{1} \in Z$.

Theorem 8.4. Assume that $(*)$ above holds. Then $A$ is a Jordan algebra if and only if $Z$ is a Jordan algebra.

In the remainder of this section we assume that $(*)$ holds.

Remark 8.5. One of our motivation for hypothesis $(*)$ above is that it holds in any primitive axial algebra of Jordan type half (not necessarily power associative). Indeed, suppose that $A$ is such an algebra. By HSS2, Theorem 4.1] $A$ admits a Frobenius form $(\cdot, \cdot)$ such that $(f, x)=\varphi_{f}(x)$, for all half-axes $f \in A$. Hence

$$
\varphi_{e}\left(u_{1}\left(u_{2} z\right)\right)=\left(e, u_{1}\left(u_{2} z\right)\right)=\left(e u_{1}, u_{2} z\right)=\frac{1}{2}\left(u_{1}, u_{2} z\right)=\frac{1}{2}\left(u_{1} u_{2}, z\right) .
$$

By symmetry, $\varphi_{e}\left(u_{2}\left(u_{1} z\right)\right)=\frac{1}{2}\left(u_{1} u_{2}, z\right)$, so indeed $(*)$ holds in $A$.

Proposition 8.6. Identity (i) of Theorem 7.2 holds, namely

$$
u_{1}\left(u_{1}^{2} u_{2}\right)=u_{1}^{2}\left(u_{1} u_{2}\right)
$$

for all $u_{1}, u_{2} \in U$.

Proof. If $u_{1}^{2} \notin Z$, this is Lemma6.3. So suppose $u_{1}^{2} \in Z$. Then $\delta_{u_{1}^{2}}=0$, so, by Theorem 1.4 $(1), u_{1}^{3}=0$. By $(*)$ we have $\varphi_{e}\left(u_{1}\left(u_{2} u_{1}^{2}\right)\right)=\varphi_{e}\left(u_{2}\left(u_{1} u_{1}^{2}\right)\right)=0$. Thus $u_{1}\left(u_{2} u_{1}^{2}\right) \in Z$. By Lemma 4.1,

$$
\left(u_{1} u_{2}\right) u_{1}^{2}=u_{1}\left(u_{2} u_{1}^{2}\right)+u_{2}\left(u_{1} u_{1}^{2}\right)=u_{1}\left(u_{2} u_{1}^{2}\right) .
$$

Proposition 8.7. Identity (ii) of Theorem 7.2 holds, namely

$$
\left(u_{1}^{2} u_{2}\right) z+2\left(u_{1}\left(u_{2}\left(u_{1} z\right)\right)=u_{1}^{2}\left(u_{2} z\right)+2\left(u_{1} u_{2}\right)\left(u_{1} z\right)\right.
$$

for all $u_{1}, u_{2} \in U$ and $z \in Z$.

Proof. Let $\delta_{1}:=\delta_{u_{1}^{2}}$. We have

$$
\begin{aligned}
& \left(u_{1}^{2} u_{2}\right) z+2 u_{1}\left(u_{2}\left(u_{1} z\right)\right) \\
& \stackrel{(i)}{=}-2 u_{1}\left(\left(u_{1} u_{2}\right) z\right)+2\left(u_{1} u_{2}\right)\left(u_{1} z\right)+2 u_{1}\left(u_{2}\left(u_{1} z\right)\right)+\delta_{1} u_{2} z \\
& =2 u_{1}\left(-\left(u_{1} u_{2}\right) z+u_{2}\left(u_{1} z\right)\right)+2\left(u_{1} u_{2}\right)\left(u_{1} z\right)+\delta_{1} u_{2} z \\
& \stackrel{(i i)}{=} 2 u_{1}\left(-u_{1}\left(u_{2} z\right)+2 \delta_{u_{2}\left(u_{1} z\right)} e\right)+2\left(u_{1} u_{2}\right)\left(u_{1} z\right)+\delta_{1} u_{2} z \\
& =-2 u_{1}\left(u_{1}\left(u_{2} z\right)\right)+2\left(u_{1} u_{2}\right)\left(u_{1} z\right)+2 \delta_{u_{2}\left(u_{1} z\right)} u_{1}+\delta_{1} u_{2} z \\
& \stackrel{(i i i)}{=} u_{1}^{2}\left(u_{2} z\right)-\delta_{1} u_{2} z-2 \delta_{u_{2}\left(u_{1} z\right)} u_{1}+2\left(u_{1} u_{2}\right)\left(u_{1} z\right)+2 \delta_{u_{2}\left(u_{1} z\right)} u_{1}+\delta_{1} u_{2} z \\
& =u_{1}^{2}\left(u_{2} z\right)+2\left(u_{1} u_{2}\right)\left(u_{1} z\right) .
\end{aligned}
$$

Where equality (i) comes from Lemma 4.4(4), equality (ii) comes from Lemma 4.1 and $(*)$. Finally, equality (iii) comes from Lemma 4.4(3). 
Proposition 8.8. Identity (iii) of Theorem 7.2 holds, namely

$$
\left(u_{1}^{2} z_{2}\right) z_{1}+2 u_{1}\left(\left(u_{1} z_{1}\right) z_{2}\right)=u_{1}^{2}\left(z_{1} z_{2}\right)+2\left(u_{1} z_{1}\right)\left(u_{1} z_{2}\right),
$$

for all $u_{1} \in U$ and $z_{1}, z_{2} \in Z$.

Proof. We use Theorem 1.4(6) and Lemma 4.1;

$$
\begin{aligned}
& \left(u_{1}^{2} z_{2}\right) z_{1}+2 u_{1}\left(\left(u_{1} z_{1}\right) z_{2}\right)=2\left(u_{1}\left(u_{1} z_{2}\right)\right) z_{1}+2 u_{1}\left(\left(u_{1} z_{1}\right) z_{2}\right) \\
& =2\left(u_{1} z_{1}\right)\left(u_{1} z_{2}\right)+2 u_{1}\left(\left(u_{1} z_{2}\right) z_{1}\right)+2 u_{1}\left(\left(u_{1} z_{1}\right) z_{2}\right)-2 \delta_{u_{1}\left(\left(u_{1} z_{2}\right) z_{1}\right)} e-2 \delta_{\left(u_{1} z_{1}\right)\left(u_{1} z_{2}\right)} e \\
& =2\left(u_{1} z_{1}\right)\left(u_{1} z_{2}\right)+2 u_{1}\left(u_{1}\left(z_{1} z_{2}\right)\right)-2 \delta_{u_{1}\left(\left(u_{1} z_{2}\right) z_{1}\right)} e-2 \delta_{\left(u_{1} z_{1}\right)\left(u_{1} z_{2}\right)} e \\
& =2\left(u_{1} z_{1}\right)\left(u_{1} z_{2}\right)+u_{1}^{2}\left(z_{1} z_{2}\right)+2 \delta_{u_{1}\left(u_{1}\left(z_{1} z_{2}\right)\right)} e-2 \delta_{u_{1}\left(\left(u_{1} z_{2}\right) z_{1}\right)} e-2 \delta_{\left(u_{1} z_{1}\right)\left(u_{1} z_{2}\right)} e .
\end{aligned}
$$

Note now that by $(*)$,

$$
\delta_{u_{1}\left(\left(u_{1} z_{2}\right) z_{1}\right)} e=\delta_{\left(u_{1} z_{1}\right)\left(u_{1} z_{2}\right)} .
$$

Hence we get

$$
\begin{gathered}
\left(u_{1}^{2} z_{2}\right) z_{1}+2 u_{1}\left(\left(u_{1} z_{1}\right) z_{2}\right) \\
=2\left(u_{1} z_{1}\right)\left(u_{1} z_{2}\right)+u_{1}^{2}\left(z_{1} z_{2}\right)+2 \delta_{u_{1}\left(u_{1}\left(z_{1} z_{2}\right)\right)} e-4 \delta_{\left(u_{1} z_{1}\right)\left(u_{1} z_{2}\right)} e .
\end{gathered}
$$

But by Lemma 4.5)(6), $2 \delta_{u_{1}\left(u_{1}\left(z_{1} z_{2}\right)\right)}=4 \delta_{\left(u_{1} z_{1}\right)\left(u_{1} z_{2}\right)}$, so we are done.

Proposition 8.9. Identity (iv) of Theorem 7.2 holds, namely

$$
u_{1}\left(u_{2} z^{2}\right)+2\left(u_{2}\left(u_{1} z\right)\right) z=\left(u_{1} u_{2}\right) z^{2}+2\left(u_{1} z\right)\left(u_{2} z\right),
$$

for all $u_{1}, u_{2} \in U$ and $z \in Z$.

Proof. We have

$$
\begin{aligned}
& u_{1}\left(u_{2} z^{2}\right)+2\left(u_{2}\left(u_{1} z\right)\right) z \\
& \stackrel{(i)}{=}\left(u_{1} u_{2}\right) z^{2}-u_{2}\left(u_{1} z^{2}\right)+2 \delta_{u_{1}\left(u_{2} z^{2}\right)} e+2\left(u_{2}\left(u_{1} z\right)\right) z \\
& \stackrel{(i i)}{=}\left(u_{1} u_{2}\right) z^{2}-2 u_{2}\left(\left(u_{1} z\right) z\right)+2 \delta_{u_{1}\left(u_{2} z^{2}\right)} e+2\left(u_{2}\left(u_{1} z\right)\right) z \\
& \stackrel{(i i i)}{=}\left(u_{1} u_{2}\right) z^{2}-2\left(\left(u_{2}\left(u_{1} z\right)\right) z-\left(u_{1} z\right)\left(u_{2} z\right)+2 \delta_{\left(u_{1} z\right)\left(u_{2} z\right)} e\right) \\
& +2 \delta_{u_{1}\left(u_{2} z^{2}\right)} e+2\left(u_{2}\left(u_{1} z\right)\right) z \\
& =\left(u_{1} u_{2}\right) z^{2}+2\left(u_{1} z\right)\left(u_{2} z\right)-4 \delta_{\left(u_{1} z\right)\left(u_{2} z\right)} e+2 \delta_{u_{1}\left(u_{2} z^{2}\right)} e \\
& \stackrel{(i v)}{=}\left(u_{1} u_{2}\right) z^{2}+2\left(u_{1} z\right)\left(u_{2} z\right)-4 \delta_{\left(u_{1} z\right)\left(u_{2} z\right)} e+4 \delta_{u_{1}\left(\left(u_{2} z\right) z\right)} e \\
& \stackrel{(v)}{=}\left(u_{1} u_{2}\right) z^{2}+2\left(u_{1} z\right)\left(u_{2} z\right) .
\end{aligned}
$$

Where equalities $(i)$ and $(i i i)$ come from Lemma 4.1 (and $(*)$ ), equalities (ii) and $(i v)$ come from the fact that $2(u z) z=u z^{2}$, and equality $(v)$ comes from $(*)$. 
Lemma 8.10. Identity (vi) of Theorem 7.2 holds, namely

$$
\left(u_{1} u_{2}\right) z+2 e\left(u_{2}\left(u_{1} z\right)\right)=u_{1}\left(u_{2} z\right)+u_{2}\left(u_{1} z\right)
$$

for all $u_{1}, u_{2} \in U$ and $z \in Z$.

Proof. This is immediate from $(*)$ and Lemma 4.1 .

Proofs of Theorems 8.1, 8.2, 8.3 and 8.4.

Theorem 8.1 is immediate from Theorem 7.2 and Propositions 8.6, 8.7, 8.8, 8.9, and Lemma 8.10, Furthermore these propositions and Lemma show that if we take $\beta=z_{2}=0$ in Theorem [7.2, then all identities in that proposition are satisfied, for all $u_{1}, u_{2} \in U$ and $z_{1} \in Z$, so Theorem 7.2 completes the proof of Theorem 8.2 (taking $y=u_{2}=u$ ). The proof of Theorem 8.3 is similar. Finally Theorem 8.4 is immediate from Theorem 8.1 and Theorem 7.2 .

Acknowledgement. The referee report of this paper can be considered as an article on its own right. It upgraded the level of this paper and improved the proofs in many parts. For example Theorem 7.1 is due to the referee. The author thinks that there is only one mathematician in the world (in areas related to this paper) that could produce such a fantastic report.

\section{REFERENCES}

[HRS] J.I. Hall, F. Rehren, S. Shpectorov, Primitive axial algebras of Jordan type, J. Algebra 437 (2015), 79-115.

[HSS1] J.I. Hall, Y. Segev, S. Shpectorov, Miyamoto involutions in axial algebras of Jordan type half, to appear in Israel J. Math. (See https://arxiv.org/abs/1610.01307.)

[HSS2] J.I. Hall, Y. Segev, S. Shpectorov, On primitive axial algebras of Jordan type, submitted. (See https://arxiv.org/abs/1705.03492.)

[M] K. McCrimmon, A taste of Jordan algebras, Universitext. Springer-Verlag, New York, 2004.

[O] J. M. Osborn, Varieties of algebras, Advances in Math. 8 (1972) 163-369.

[TB] D. A. Towers, K. Bowman, On power associative Bernstein algebras of arbitrary order, Algebras Groups Geom. 13 (1996), no. 3, 295-322.

Yoav Segev, Department of Mathematics, Ben-Gurion University, BeerSHEVA 84105, ISRAEL

E-mail address: yoavs@math.bgu.ac.il 